\title{
Linx
}

Revue des linguistes de l'université Paris X Nanterre

12 | 2002

«Comme la lettre dit la vie »

\section{Prospectualisation analeptique dans le Roman de Mélusine de Jean d'Arras}

\section{Sara Sturm-Maddox et Donald Maddox}

\section{OpenEdition}

\section{Journals}

Édition électronique

URL : http://journals.openedition.org/linx/1298

DOI : 10.4000/linx.1298

ISSN : 2118-9692

\section{Éditeur}

Presses universitaires de Paris Nanterre

\section{Édition imprimée}

Date de publication : 1 octobre 2002

Pagination : 173-177

ISSN : 0246-8743

\section{Référence électronique}

Sara Sturm-Maddox et Donald Maddox, « Prospectualisation analeptique dans le Roman de Mélusine de Jean d'Arras », Linx [En ligne], 12 | 2002, mis en ligne le 10 octobre 2012, consulté le 02 mai 2019. URL : http://journals.openedition.org/linx/1298; DOI : 10.4000/linx.1298

Ce document a été généré automatiquement le 2 mai 2019.

Département de Sciences du langage, Université Paris Ouest 


\title{
Prospectualisation analeptique dans le Roman de Mélusine de Jean d'Arras
}

\author{
Sara Sturm-Maddox et Donald Maddox
}

1 L'analepse fait partie de l'organisation transphrastique du texte narratif et nous amène donc bien au-delà d'une linguistique pour ainsi dire «logométrique ». Or notre propos dans ce qui s'ensuit est de montrer comment, en dotant la fiction d'un passé - que ce soit d'un passé certain, ou simplement d'un certain passé - l'analepse en vient parfois, et souvent de façon quelque peu paradoxale, à remplir des fonctions qui sont plutôt proleptiques, en convoquant la suite du récit en cours d'élaboration. Ainsi en est-il des instances de ce que nous nous proposons d'appeler la prospectualisation analeptique qui viennent rythmer le parcours narratif du Roman de Mélusine de Jean d'Arras.

2 Dans ce roman, c'est par moyen d'analepses que les principaux personnages masculins de la dynastie des Lusignan prennent connaissance de leur propre lignage paternel ou maternel. De sa femme Mélusine Raimondin apprend l'identité et le passé du père qui lui avait été jusqu'alors inconnu. Bien plus tard Geoffroy, celui parmi leurs fils qui sera l'héritier de Lusignan, découvre au cours d'une aventure singulière le passé lignager de Mélusine $^{1}$. Or à la seconde de ces révélations lignagères viennent s'ajouter deux autres scènes où l'analepse, quoique moins dramatique et moins développée, s'avère lourde de conséquences. S'enchaînant à l'aventure de Geoffroy, elles forment un ensemble qui ouvre la voie à une réorientation fondamentale de la suite et fin du récit.

3 Lorsque Mélusine raconte la vie d'Hervé à Raimondin, le narrateur lui cède la parole, comme si elle seule-par qui sait quel pouvoir féerique? - connaissait cette histoire. A la différence de cette analepse hétérodiégétique, celles par lesquelles Geoffroy découvre le passé de sa mère font partie de la diégèse antécédente ${ }^{2}$. D'où en fait deux variétés d'analepse : tandis que l'une conditionne l'avenir - la démarche par laquelle Raimondin, sous la tutelle de Mélusine, s'emparera de l'héritage breton qui lui revient, l'autre éveille, d'abord chez Geoffroy, puis chez Raimondin, une prise de conscience capitale.

Parmi les nombreux fils de Mélusine et Raimondin, seule s'accorde à Geoffroy, racontée à intervalles dans le roman, une biographie quasi intégrale ${ }^{3}$. Dans la postface à sa traduction en français moderne du Mélusine de Jean d'Arras, Michèle Perret a bien 
formulé le rôle critique de ce fils par rapport au passé et à l'avenir de Lusignan : « Or c'est à ce personnage presque sans mémoire qu'incombe d'assumer tout le poids de l'histoire familiale: découvrir la faute de la mère, retrouver et réconforter le père, délivrer Lusignan $»^{4}$. Toutefois le rôle de Geoffroy, avant qu'il ne réalise ces exploits, paraît fort ambigu. De tous les fils de Mélusine qui portent les tares de leur héritage féerique, c'est lui qui fait preuve des tendances les plus violentes. Personnage presque surhumain par sa force et par son courage, il se distingue aux entreprises politico-militaires des frères Lusignan en Orient : s'il ne réussit pas à prendre Jérusalem, il met sa prouesse au service de Dieu et gagne l'amitié du sultan de Damas ${ }^{5}$. En revanche, ses cruautés et sa démesure semblent être programmées dès sa naissance par la grande dent saillante pour laquelle on l'avait nommé . Sans doute l'ambivalence du personnage s'explique-t-elle en partie par des réminiscences historiques, soit de Geoffroy I de Lusignan, guerrier renommé, soit surtout de Geoffroy II, tueur de moines, enfin excommunié 7 . En outre, ce sixième fils de Lusignan semble partager avec sa mère des affinité significatives. Comme elle, il participe au « double registre mythique » dont parle Laurence Harf-Lancner : « Tueur de géants, et à ce titre figure de héros civilisateur, [Geoffroy] relève également du monde sauvage par sa fureur guerrière, qui lui fait mettre le feu à l'abbaye de Maillezais » parce que son frère Fromont s'y était fait moine ${ }^{8}$.

Personnage à double nature, donc, sinon diabolique du moins problématique ou paradoxal ${ }^{9}$. Après l'incendie meurtrier, son père met en cause Mélusine : «Par la foy que je doy a Dieu, je croy que ce ne soit que fantosme de ceste femme, ne ne croy pas que ja fruit qu'elle ait porté viengne a perfection de bien $\gg(\mathrm{M} \mathrm{253})^{10}$. Il y revient dans sa dénonciation publique de son épouse : «Hee, tres faulse serpente, par Dieu, ne toy ne tes fais ne sont que fantosme, ne ja hoir que tu ayes porté ne vendra a bon chief en la fin... [Fromont] est destruit par l'art demoniacle, car tous ceulx qui sont forcennez de yre sont ou commandement des princes d'enfer" (M 255). Accusation lourde des conséquences que l'on sait pour la fée-épouse, obligée - malgré son gré et en dépit des repentirs de Raimondin - de quitter Lusignan.

Quel avenir donc pour ce fils aussi coupable que cruel ? D'après son père, il est perdu : «Haa, ce dist il, Gieffroy, or avoies tu le plus bel commencement de prouesse et de bachelerie pour venir ou degré de haulte honneur, que filz de prince qui feust vivans. Et ores en est tu du tout desmis par ta cruauté » (M 253). Certes, le statut même de Geoffroy, comme celui des autres fils de Mélusine, peut le rendre suspect : selon Gautier Map, parmi d'autres autorités cléricales, les enfants nés de l'union d'un homme mortel avec une fée sont voués à une mauvaise fin ${ }^{11}$. Par contre, Mélusine fait remarquer à son époux que la perdition n'est pas inéluctable : "Sachiez qu'il n'a si grant pecheur ou monde que Dieu ne soit plus grant pardonneur et plus debonnaire, quant le pecheur se repent et lui crie mercy de bon cuer et de bonne voulenté » (M 255). En plus, si elle suggère que Geoffroy aurait agi en agent de Dieu pour punir le "pechié des moines, qui estoient de mauvaise vie et desordonnee...les jugemens de Dieu sont si secrez que nul cuer mondain ne les puet comprendre en son entendement » (M 255) - sa perception du jugement divin cadre bien avec celle du prologue du roman. Qui a raison, Raimondin ou Mélusine? De la réponse dépend non seulement le salut de Geoffroy mais l'avenir de la dynastie, dont Mélusine, avant son départ, lui exprime toute sa confiance qu'il en assurera la survie. C'est à lui que Raimondin doit laisser la disposition des terres de Lusignan qu'elle vient de décréter aux autres fils. «Et en laissiez convenir a Gieffroy, » lui dit-elle, « car il en ordonnera bien » (M 258). A ce stade, le statut de Geoffroy reste en suspens. Et pourtant l'image négative, 
dressée auparavant par Jean d'Arras, de ce fils semble s'éclipser pour faire désormais place à une tentative de le récuperer pour la gloire de son lignage ${ }^{12}$.

7 Or, c'est au service de cette récupération que la série d'analepses qui s'ensuit acquiert une importance capitale. En fait, Geoffroy est d'emblée envahi de remords devant le spectacle de son crime : en contemplant l'incendie, il entrevoit «le meschief et le dommage qu'il avoit fait. Lors se plaint et se guermente et se nomme faulx et mauvais, et se dit tant de laidure qu'il n'est homs qui le peust penser s'il ne le veoit ou ouoit. Et croy que de fin ennuy il se feust occiz de l'espee, se ne feust que les x. chevaliers y vindrent» (M 252). Peu de temps après, en poursuivant un géant rebelle à l'autorité de Raimondin, il s'introduit aux profondeurs d'une montagne et y découvre une chambre sépulcrale; sur la tombe se trouve le gisant d'un roi, à ses pieds l'image sculptée d'une reine, qui tient un «tablel ». L'histoire y inscrite, résumant le premier volet du roman, n'offre au lecteur rien de nouveau: le mariage d'Elinas, roi d'Albanie, avec la fée Présine; l'interdit que celle-ci lui avait imposé et qu'il avait enfreint ; la disparition de Présine avec leurs trois filles, dont Mélusine ; la révélation de la perfidie du père et la revanche que les filles en avaient prise, en enfermant le roi pour toujours dans la montagne; enfin le sort que Présine avait imposé à chaque fille en tant que punition. Le narrateur se contente donc d'évoquer sommairement les détails de ce lointain passé : il affirme que le tablel « devisoit toute la maniere comment » ces choses extraordinaires sont advenues et que l'histoire s'y trouve toute entière : «tout au long, ainsi qu'il est escript cy dessus ou chapitre du roi Elinas » (M 266).

8 Tout en rappelant au lecteur ce qu'il a déjà lu, ce stratagème narratif sert à focaliser l'attention sur Geoffroy, qui lit maintenant dans le détail une histoire qu'il a jusqu'ici ignorée. Toutefois, il n'arrive pas à s'y reconnaitre : «Lors musa Gieffroy grant temps, tant sur le tablel comme sur la beauté du lieu; mais encore ne scot il pas qu'il soit de la lignee du roy Elinas et de Presine » (M 266). La chaîne de communication lignagère sera bientôt bouclée quand un de ses frères l'informe que leur mère a définitivement quitté Lusignan. De cette histoire aussi, le lecteur sait déjà ce que Geoffroy apprend pour la première fois: provoquée par la destruction de l'abbaye par Geoffroy, la fureur de Raimondin, qui déclare publiquement avoir vu la transformation serpentine de sa femme; le départ de celle-ci sous une forme monstrueuse. Encore une fois, le narrateur abrège l'aperçu rétrospectif en cumulant les «comment »: le frère raconte à Geoffroy «toute la maniere et la guise, du commencement jusques en la fin, comment leur mere estoit partie, et toute l'adventure, et comment elle avoit dit, au partir, qu'elle estoit fille au roy Elinas d'Albanie » (M 268). Ce dernier élément, pourtant, renvoie aussi, sans que le frère le sache, à l'aventure toute récente de Geoffroy, dont celui-ci saisit enfin le sens : la vérité de la parole maternelle, ici rapportée, vient confirmer le témoignage oculaire que Geoffroy vient de faire à l'intérieur de la montagne.

9 Ainsi se reconnaît-il enfin dans le personnage du lignage d'Elinas et de Présine prévu sur le «tablel », celui destiné à tuer le géant gardien du somptueux tombeau royal. « Gieffroy entendy ces mos, si lui souvint du tablel qu'il avoit trouvé sur la tombe du roy Elinas, et lors scot au cler qu'ilz estoient il et ses freres, descenduz de sa lignie, si s'en tint plus chiers » (M 268). Reconnaissant ainsi son appartenance, du côté maternel, à cette lignée royale, maintenant il doit également reconnaître sa part, d'ailleurs considérable, de responsabilité dans le triste sort de ses parents, et le narrateur fait état chez ce personnage d'habitude si «féroce » et si «cruel » d'un émoi tout nouveau : «il fu moult doulent de la perte de sa mere et de la douleur de son pere » (M 268). Il est vrai que dans 
l'immédiat, il semble récidiver: ayant appris que son oncle avait provoqué chez Raimondin la violation de l'interdiction de Mélusine, il se lance à sa poursuite, et l'oncle périt en tombant du haut de son château (M 268-69). Averti de ce décès, Raimondin croit retrouver chez son fils sa nature inconsidérée et violente d'autrefois. «Il me fault appaisier a Gieffroy avant qu'il face plus de dommages », conclut-il, et il convoque son fils à Lusignan. Lorsque Geoffroy arrive, pourtant, il se jette aux pieds du père et lui implore pardon, jurant de reconstruire l'abbaye qu'il avait détruite (M 269).

Mais s'agit-il en vérité du repentir ? Quant à Raimondin, il ne fait que rappeler à son fils la gravité de ses méfaits : «Par foy, dist Remond, tout ce se puet bien faire, mais aux mors ne povez vous rendre la vie » (M 269). En revanche, il annonce qu'il laisse à Geoffroy le gouvernement de Lusignan, aussi bien que la disposition de ses autres terres selon la volonté de Mélusine, pour partir lui-même en pèlerinage. A son départ, Geoffroy et un de ses frères l'accompagnent, et chemin faisant Geoffroy leur fait part de sa découverte du tombeau dans la montagne et de ce qu'il y avait appris. Il raconte «toute la besoingne, » dit le narrateur, « ainsi comme elle fu, et que je l'ay retraicte ou chappitre du roy Elinas, ou commencement de ceste histoire» (M 270). Pas de commentaire, ni de la part de Geoffroy, narrateur intradiégétique, ni de la part du narrateur du roman. A quoi sert donc la reprise de cette scène déjà bien connue du lecteur?

11 Cette fois le destinataire principal en est Raimondin. « Et sachiez, » dit le narrateur, « que Remond l'escouta diligemment et l'ouy tres voulentiers, et lui plot moult, car Gieffroy leur afferme que sa mere fu fille du roy Elinas et de Presine » (M 270). Réaction curieuse, en fait : on sait que Mélusine avait tout récemment souligné cette filiation, juste avant son départ de Lusignan, en faisant ses adieux : «Et toutesfoiz je vueil bien que vous sachiez qui je sui ne qui fu mon pere, afin que vous ne reprouvez pas a mes enfans qu'ilz soient filz de mauvaise mere, ne de serpente, ne de faee, car je suiz fille au roy Elinas d'Albanie et a la royne Presine, sa femme, et sommes iij. seurs qui avons esté durement predestinees et en griefz penitances » (M 259-60). D'après cette réaction de Raimondin au récit de son fils, on s'aperçoit qu'il avait toujours nourri des doutes, après le départ de Mélusine, concernant la nature "merveilleuse » de celle-ci. Sans toutefois que son amour ne diminue : ne l'avait-il pas épousée tout en sachant qu'elle n'était pas « une femme comme toutes les autres »? Mais ni l'apaisement de sa colère, ni la douleur de l'avoir perdue n'avaient annulé les jugements sévères qu'il avait portés sur elle. L'émoi de Raimondin devant le triste spectacle de l'abbaye incendiée - «Je croy que ce ne soit que fantosme de ceste femme » (M 253) - rappelle l'assurance que Mélusine lui avait offerte avant leur mariage: "...je scay bien que tu cuides que ce soit fantosme ou euvre dyabolique de mon fait et de mes paroles, mais je te certiffie que je suiz de par Dieu et croy en tout quanque vraye catholique doit croire » $(\mathrm{M} 25)^{13}$. Il avait pleuré le départ de sa femme, mais seul le témoignage de Geoffroy semble le convaincre que l'origine de Mélusine fut légitime - royal et non monstrueux ${ }^{14}$. D'où une analepse hautement véridictoire, et qui programme la suite de son histoire.

Dès ce grant tournant dans le roman, le destin lie ces deux hommes, désormais conscients de leur part de culpabilité à l'égard du départ de Mélusine. Par où ils se ressemblent, car Geoffroy, fils de la fée, est également, par ses emportements et son impétuosité de naguère, fils de Raimondin ${ }^{15}$. Son outrance en apprenant que son frère Fromont s'est fait moine- « Il fu si doulens que a pour pou qu'il n'yssoit hors de son sens. Et sachiez qu'il sembloit mieux forsenné que autre » (M 250) ${ }^{16}$ - fait penser à celle de Raimondin lorsqu'il maudit Mélusine. Quand elle essaie de l'apaiser, le narrateur affirme qu'il sait bien 
« qu'elle lui dit voir de quanqu'elle lui avoit dit, et que c'est le meilleur selon raison. Mais il fu si tresperciez et oultrez de yre que raison naturelle s'en estoit fuye de lui » (M 255) ${ }^{17}$.

A l'endroit où le père et le fils se séparent après la disparition de Mélusine, ces deux analepses révélatrices infléchissent le sens du destin de chacun. En plus, ces rappels servent à impliquer ce qui n'est jamais explicité dans le texte concernant les états d'âme qui sous-tendent le comportement de chaque homme. Raimondin se rend à Rome, où il se confesse « au mieux qu'il pot » au Saint Père qui, du fait « qu'il s'estoit parjurez envers sa femme...lui charga tel penitence qu'il lui plot» (M 270). Résolu à devenir ermite, pour prier Dieu «qu'il lui plaise faire allegement a ma moiller », Raimondin repart avec la bénédiction du Pape pour mener «moult saincte vie » (M 271, 273), et le roman raconte ensuite son ascèse spirituelle jusqu'à sa mort. Quant à Geoffroy, de retour à Lusignan, il fait ses premières preuves de bonté : les barons que Raimondin avait instruits de rendre désormais hommage à ce fils, «moult redoubtoient Gieffroy pour sa fierté ; mais pour neant le doubtent, car il les gouvernera bien et doulcement » (M 274). En fait, la suite du roman se consacre en partie à démontrer que Geoffroy n'est pas, comme l'avait prononcé son père outré, «du tout desmis ». Autrefois "personnage presque sans mémoire, » désormais il n'oublie pas :

...moult fut doulent de ce qu'il ot par son pechié ainsi perdu son pere et sa mere... Dont remort conscience a Geiffroy, et lui souvint comment il avoit ars les moynes de Maleres, et l'abbé, et son frere Fromont, sans raison, et que par ce pechié avoit esté sa mère perdue. Puis lui ramembre de son oncle le conte de Forests, lequel il fist saillir de la grosse tour de Marcelli le Chastel sur la roche, et le fist tuer. Lors commenca Gieffroy fort a penser a ses pechiez. Et dist bien que, se Dieu n'a pitié de lui, l'ame de lui est en grant peril et en voye de dampnacion. Lors entra Gieffroy en une chambre, et commence a mener grant doulour et a plourer ses pechiez. Et la lui prist devocion d'aler a Romme confesser au Saint Pere. (M 274)

" Moult devotement, » tel son père, il se rend au Saint-Siège et se confesse au Pape qui lui impose plusieurs pénitences, et notamment celle de faire reconstruire l'abbaye de Maillezais. Mélusine n'avait-elle pas dit que l'on pourra faire reconstruire l'abbaye et que « Gieffroy s'amendera, se plaist a Dieu et au monde » (M 255) ? Il rendra pieusement visite à Raimondin ermite jusqu'au trépas de ce dernier.

La fin du Roman de Melusine en vers par Coudrette, œuvre presque contemporaine, souligne le progrès admirable de Geoffroy dans un épisode qui ne figure pas dans le roman en prose. Palestine, une des deux soeurs de Mélusine, est enfermée par sa mère dans le Mont Canigou en Aragon, où elle garde le trésor de son père Helinas par ordre de sa mère. «Et qui conquester le pourroit / Promission [la Terre Promise] en conquerroit, / Maiz jamaiz ne le conquerra / Qui de la lignie ne venra $»^{18}$. Après que maint bon chevalier tente l'aventure et y échoue, Geoffroy s'y apprête, mais il tombe malade et meurt. Coudrette loue le héros défunt : «Helas! Il eüst le tresor / Conquis, s'il eust vescu encor, / Et terre de Promission / Qui tant est saint region... / De quoy ce fut moult grant douleur, / Car en Poitou eust fait assez / De biens ains que l'an fust assez.../ Car il fist de biens grant foyson / Si tost qu'en lui se mist raison ${ }^{19}$. Jean d'Arras aussi, dans l'épilogue de son roman, lui réserve une dernière épreuve: héritier du château de Lusignan, il va défendre l'intégrité du patrimoine. Dix ans après le décès de son père, il apprend que, du vivant de Raimondin, une redevance sur la grande tour avait été imposée, et que Raimondin l'avait réglée, en tant que pénitence pour avoir parjuré son vœu à Mélusine. Ayant refusé cette imposition, Geoffroy se trouve dans l'obligation de lutter contre l'instigateur de cet usage, le mystérieux «Chevalier de la 
Tour ». Après un rude combat à la fin duquel « on ne scet gaires lequel en a le meilleur » (M 300), le chevalier s'explique: il avait institué la redevance au profit de l'âme de Raimondin, qui de son vivant n'avait pas entièrement satisfait la pénitence imposée par le Pape « pour le parjur qu'il avait fait » (M 300) à Mélusine. Maintenant il reconnaît avoir mis Geoffroy à l'épreuve, le tient quitte, et lui offre un conseil : de «faire fonder un hospital et amortir une chappellerie " pour l'âme de Raimondin (M 300). Ce qu'il ne manque pas de faire, pour compenser la transgression de son père. Grace donc à Geoffroy, le domaine de Lusignan est ainsi réconcilié avec un surnaturel, mystérieux certes, mais certes chrétien ${ }^{20}$.

Sans la trahison de Raimondin, Mélusine aurait pu, en remplissant les conditions établies par sa mère-fée, échapper à son sort monstrueux en se forgeant un destin de «femme naturelle » : juste avant son ultime métamorphose serpentine, elle se lamente que, parce que le trépas humain lui est nié, elle ne recevra pas les sacrements et n'aura pas de sépulture dans l'église de Lusignan. Suite à la prise de conscience chez Geoffroy et Raimondin, on va insister - dans cette histoire qui célèbre la maison de Lusignan - sur le fait qu'à ses deux hommes responsables de la perte de l'épouse-mère-fondatrice est réservé un meilleur sort.

18 A la lumière de ces analyses, on s'aperçoit comment, dans le Roman de Mélusine de Jean d'Arras, l'analepse se montre à plusieurs reprises capable de virtualiser la suite du récit, ce qui s'effectue par l'attribution d'un savoir dont la spécularité rétrospective vise le narrataire et par là le reconstitue en agent du faire. Malgré le fait que la dimensionnalité du récit analeptique est le plus souvent transphrastique, il est évident qu'en tant que composante qui prospectualise le discours, l'analepse est fort susceptible de receler une portée illocutoire, qu'elle soit explicite ou simplement implicite, que l'on pourrait facilement synthétiser en tant qu'énoncé à teneur performative exprimant, par exemple, une injonction, un mandat, la sanction positive d'une compétence, etc. La prospectualisation analeptique peut donc servir à la constitution d'un sujet du faire par l'attribution d'une compétence cognitive préalable à ses opérations ultérieures.

Toutefois, comme nos exemples nous permettent de constater, ce processus peut rester inachevé à l'échelle du seul récit analeptique, ce qui nous amène à proposer une distinction entre deux types de prospectualisation analeptique, l'une "perfective », où l'attribution cognitive du récit rétrospectif suffit tout seul à conditionner le faire du narrataire, l'autre « inchoative », où l'insuffisance d'ordre cognitif ou illocutoire du récit rétrospectif diffère la " perfection » du processus censé amener le narrataire au seuil de la factitivité. Est donc perfective la biographie d'Hervé de Lion narrée sous forme d'analepse par Mélusine, car elle décerne ainsi à Raymondin une compétence qui est à la fois cognitive et factitive, en annexant à son récit un mandat prescriptif destiné à constituer son époux directement en sujet du faire. Par contre, quand Geoffroy accède à la chambre mortuaire de ses ancêtres mélusiniens et lit le message spéculaire qui lui est destiné et qui l'y attend de longue date, la portée virtualisante de l'analepse n'est qu'inchoative puisque Geoffroy reste à ce stade encore incapable de saisir la visée spéculaire du message. Comme on vient de voir, il a fallu encore d'autres aperçus rétrospectifs pour qu'il parvienne enfin à se situer par rapport au spectacle souterrain. Ainsi Jean d'Arras construit-il progressivement la compétence cognitive de celui parmi les fils de Mélusine et Raymondin qui prendra la relève à la tête de la dynastie des Lusignan, et le fait de différer chez Geoffrey la pleine compréhension de sa place au sein du passé matriligne sert à montrer qu'il a enfin dépassé le stade d'impétuosité irréfléchie 
qui avait si souvent marqué négativement ses exploits de «jeune». Quant à Raymondin, l'affirmation concernant Mélusine qu'il entend dans le récit de Geoffroy l'amènera à une longue pénitence et à l'ascèse spirituelle. D'où enfin deux suites du récit qui nous permettent d'identifier, parmi les fonctions de l'analepse, celle de l'aspectualisation pour ainsi dire « anaproleptique » du personnage de fiction ${ }^{21}$.

\section{NOTES}

1. Voir Donald Maddox, «Configuring the Epilogue : Ending and the Ends of Fiction in the Roman de Mélusine, " in Melusine of Lusignan, Founding Fiction in Late Medieval France, éd. Donald Maddox et Sara Sturm-Maddox (Athens, GA : University of Georgia Press, 1996), p. 278-79.

2. Selon la typologie d'anachronies proposée par Gérard Genette, ces deux types correspondent, à quelques nuances près, l'un à l'analepse complétive, ou « renvoi, » l'autre à l'analepse répétitive, ou « rappel ». Voir Figures III (Paris : Editions du Seuil, 1972), p. 92-96.

3. Dans ce sens, le remanieur du XVI ${ }^{\mathrm{e}}$ siècle qui sépare les aventures de Geoffroy pour en faire un roman complémentaire à celui centré sur Mélusine «ne faisait que pousser à son terme une tendance déjà marquée dans le roman médiéval. » Voir Laurence Harf-Lancner, «Le Roman de Mélusine et le Roman de Geoffroy à la grand dent: les éditions imprimées de l'œuvre de Jean D’Arras, » Bibliothèque d'Humanisme et Renaissance, 50 (1988), p. 366.

4. Jean d'Arras, Mélusine, trad. Michèle Perret (Paris : Stock Plus, 1979 ; réimpr. 1992), p. 331.

5. Comme le remarque Emmanuèle Baumgartner : «In sum, an inventory of 'conquests' that lend the monstrous son of the serpent-woman an aura of the sacred, the prestige of a Godfrey of Bouillon, indeed of the legendary Charlemagne of the Voyage à Jérusalem et à Constantinople.» «Fiction and History: The Cypriot Episode in Jean d'Arras's Mélusine, » dans Melusine of Lusignan, p. 193.

6. Pour le côté négatif du personnage voir S. Roblin, «Le sanglier et la serpente : Geoffroy la Grant'Dent dans l'histoire des Lusignan, » dans Métamorphose et bestiaire fantastique au Moyen Age, éd. Laurence Harf-Lancner (Paris: Collection de l'Ecole Supérieure de Jeunes Filles, 1985), p. 247-85 ; Gabrielle M. Spiegel, « Maternity and Monstrosity : Reproductive Biology in the Roman de Mélusine, » dans Melusine of Lusignan, p. 109-10 ; Stephen G. Nichols, « Melusine Between Myth and History : Profile of a Female Demon, » dans Melusine of Lusignan, p. 144-49.

7. Voir Coudrette, Le roman de Mélusine, trad. Laurence Harf-Lancner (Paris : GF-Flammarion, 1993) : " Geoffroy I ${ }^{\text {er }}$ devait être l'un des héros de la troisième croisade. Quant à Geoffroy II, qui revendiquait des privilèges sur les domaines de l'abbaye de Maillezais, il tua quelques moines, incendia l'abbaye, fut excommunié et dut, en 1233, solliciter du pape son absolution » (p. 26-27).

8. Harf-Lancner, Le roman de Mélusine, p. 23-24.

9. Pour la conduite problématique de Geoffroy voir Douglas Kelly, «The Domestication of the Marvelous in the Melusine Romances" dans Melusine of Lusignan, p. 34-35, 43, et sur le côté paradoxal de son comportement, voir Rupert T. Pickens, «The Poetics of Paradox in the Roman de Mélusine » dans Melusine of Lusignan, pp. 60-61, 65-67.

10. Jean d'Arras, Mélusine. Roman du XIVe siècle, éd. Louis Stouff (Genève : Slatkine Reprints, 1974). 11. Spiegel, "Maternity and Monstrosity,» p. 111 et note 30 ; c'est cette "inherently bad biology », propose-t-elle, qui expliquerait la tragédie de cette famille. 
12. Jane H. M. Taylor, « Melusine's Progeny : Patterns and Perplexities, » dans Melusine of Lusignan ,p. 177.

13. Voir à ce sujet Gabrielle Spiegel, « Maternity and Monstrosity, » p. 111.

14. La comparaison s'impose avec le «principe de la signification différée ou suspendue » étudié par Genette chez Proust, dans A la recherche du temps perdu (voir Figures III, p. 97).

15. Voir les propos de Laurence de Looze, «'La fourme du pié toute escripte' : Melusine and the Entrance into History, » dans Melusine of Lusignan, p. 125-36.

16. Le messager qui apporte la nouvelle à Raimondin l'explique ainsi : «Gieffroy au grant dent, vostre filz, a prins telle merancolie et tel dueil de ce que... » etc. (M 252).

17. En ce qui concerne sa perte de 'raison naturelle', voir Donald Maddox, Fictions of Identity in Medieval France (Cambridge, England: Cambridge University Press, 2000), p. 180.

18. Coudrette, Le roman de Mélusine ou histoire de Lusignan, éd. Eleanor Roach (Paris : Klincksieck, 1982), v. 6229-32.

19. V. 6613-16; 6628-30; 6665-66. L'importance de cette histoire, qui relie Geoffroy de Lusignan à la reconquête de la Terre Sainte, est soulignée par L. Harf-Lancner dans l'introduction à sa traduction du roman de Coudrette, p. 34.

20. Maddox, « Configuring the Epilogue, » p. 269-73, 279 ; pour la réfutation de l'attribution d'une identité diabolique au chevalier mystérieux, voir p. 273-74.

21. Soulignons le fait que toutes les analepses qui figurent dans ce roman ne fonctionnent pas ainsi. A titre d'exemple, citons celle, finement analysée par Michèle Perret (Mélusine, p. 327-30), qui renvoie à l'image féerique, fugitive, de celle qui avait assuré la réussite des exploits foréziens du père de Raimondin (éd. Stouff, p. 15). Ainsi s'ouvre l'abyme d'un passé insondable, dont les implications sont en fait assez troublantes.

\section{AUTEURS}

\section{SARA STURM-MADDOX}

University of Massachusetts, Amherst

\section{DONALD MADDOX}

University of Massachusetts, Amherst 